\title{
Refractory hyperkalemia caused by ACE gene mutation in a 3-year- old girl with kidney transplantation: a case report
}

\author{
Jeong Yeon Kim', Beom Hee Lee ${ }^{2}$, Heeyeon Cho \\ ${ }^{1}$ Division of Nephrology, Department of Pediatrics, Samsung Medical Center, Seoul, Korea \\ ${ }^{2}$ Department of Pediatrics, Asan Medical Center, University of Ulsan College of Medicine, Seoul, Korea
}

Background: Renal tubular dysgenesis (RTD) is rare and mostly fatal disease caused by genetic defect in renin-angiotensin system (RAS) presenting poor or absent development of proximal tubule. We report a child with non-fatal clinical course who was confirmed as RTD after kidney transplantation (KT).

Methods: Medical record of a child who diagnosed as RTD with ACE gene mutation after KT was retrospectively reviewed. The whole exome sequencing was performed to confirm genetic mutation with DNA extracted from saliva.

Results: In this patient, severe oligohydramnios was detected at gestation age of 17 weeks and due to persistent oligohydramnios her mother went emergency cesarean section at the 32 weeks of gestation. After a week after birth, diuretic resistant oliguria developed and continuous renal replacement therapy was applied for a week. Although urination recovered, renal insufficiency persisted. At the age of 7 months, peritoneal dialysis was initiated. At the age of 3 years, she received a KT from a deceased donor. Immediately after surgery, she had no complication and discharged. A week after discharge, severe hyponatremia, hyperkalemia, azotemia with metabolic acidosis was detected with polydipsia and polyuria. After intravenous hydration with normal saline and kalimate administration, laboratory values were adjusted. This event was repeated twice more, and polyuria was considered the cause. Nephrectomy for both native kidney was done at 80 days after KT to control polyuria. Nevertheless, electrolyte imbalance, metabolic acidosis, and azotemia persisted. To verify the reason for hyperkalemia, renin activity and aldosterone was evaluated and renin activity was increased $(25.33 \mathrm{ng} / \mathrm{mL} / \mathrm{hr})$ with decreased aldosterone level $(2.6 \mathrm{ng} / \mathrm{dL})$. Fludrocortisone was added and laboratory values were improved. She underwent whole exome sequencing to verify cause of hypoaldosteronism and homozygous ACE gene mutation (p.Ser486PhefsTer29) was revealed.

Conclusions: As RTD can be presented in non-fatal form, RAS defect should be considered in KT patients with refractory hyperkalemia.

Corresponding author: Jeong Yeon Kim

E-mail:kim.jy1117@gmail.com

(c) The Korean Society for Transplantation

This is an Open Access article distributed under the terms of the Creative Commons Attribution Non-Commercial License (http://creativecommons.org/licenses/by-nc/4.0/) which permits unrestricted non-commercial use, distribution, and reproduction in any medium, provided the original work is properly cited. 